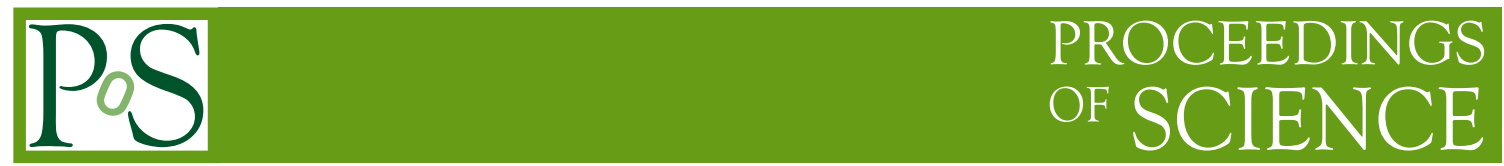

\title{
Dynamical Gauge Symmetry Breaking in Strongly Coupled Lattice Theories
}

\section{Simon Catterall}

Department of Physics, Syracuse University

E-mail: smcatterallephysics.syr.edu

\section{Aarti Veernala*}

Department of Physics, Syracuse University

E-mail: aveernalesyr.edu

We show how a strongly coupled lattice theory consisting of just fermions and gauge fields can exhibit a dynamical Higgs mechanism through the formation of a gauge invariant four fermion condensate. Furthermore, we argue that the resultant lattice Higgs phase may survive into the continuum limit.

31st International Symposium on Lattice Field Theory - LATTICE 2013

July 29 - August 3, 2013

Mainz, Germany

\footnotetext{
* Speaker.
} 


\section{Introduction}

The idea that the Higgs mechanism can occur through the formation of fermionic condensates is an attractive one when constructing many theories of Beyond Standard Model (BSM) physics and finds application in technicolor, composite Higgs models, tumbling and grand unification schemes $[1,2,3,4]$. Lattice realizations of these scenarios thus potentially give a rigorous setting for understanding how non-perturbative dynamics in models without elementary scalars can spontaneously break gauge symmetries and potentially can give us new tools to analyze such theories.

In a lattice theory, Elitzur's theorem [5] guarantees that any condensate which is not invariant under the gauge symmetry must necessarily have vanishing expectation value. Instead, the gauge invariant way to understand the operation of the Higgs mechanism in such theories is that it proceeds via the condensation of a gauge invariant four fermion operator. In the models we will consider the gauge interactions factorize into strong and weak sectors. The Higgsing of the weak gauge symmetry will be signaled by the appearance of a non-zero vacuum expectation for a four fermion operator of the form $\phi^{\dagger} \phi$ where $\phi$ is a composite Higgs field which is a singlet under the strong interaction but is charged under the weak group.

In section 2, we start our construction by considering two independent massless staggered fermions. Each of these fields can be projected (reduced) so that one of them is only defined on even parity lattices sites while the other resides on the odd lattice sites. The key observation is that the kinetic terms for each of these reduced staggered fermions may be gauged independently from each other. However, as a consequence, single site fermion bilinear terms will, in general, break gauge invariance. We use this feature to build interesting models in which the strong interactions force the formation of condensates which Higgs the weakly coupled symmetries. We consider several examples of this symmetry breaking scenario for both global and local symmetries and show preliminary numerical results which are consistent with these arguments.

\section{Lattice Model and Gauge Symmetry Breaking}

We start with two staggered fields $\chi$ and $\xi$. After restricting them to odd/even sites, we can define new fields $\psi$ and $\lambda$ as :

$$
\begin{aligned}
\bar{\psi}_{+}(x) & =\frac{1}{2}(1+\varepsilon(x)) \bar{\chi}(x), & \bar{\lambda}_{-}(x) & =\frac{1}{2}(1-\varepsilon(x)) \bar{\xi}(x) \\
\lambda_{+}(x) & =\frac{1}{2}(1+\varepsilon(x)) \xi(x), & \psi_{-}(x) & =\frac{1}{2}(1-\varepsilon(x)) \chi(x)
\end{aligned}
$$

where the parity of a lattice site is given by $\varepsilon(x)=(-1)^{\sum_{\mu=1}^{4} x_{\mu}}$. The fields $\psi$ and $\lambda$ are termed reduced staggered fermions since each contains half the number of degrees of freedom of the usual staggered fermion and corresponds to two rather than four Dirac fermions in the continuum limit $[7,8]$. The resultant lattice action can then be written as,

$$
S=\sum_{x, \mu} \eta_{\mu}(x) \bar{\psi}_{+}(x)\left(\psi_{-}(x+\mu)-\psi_{-}(x-\mu)\right)+\sum_{x, \mu} \eta_{\mu}(x) \bar{\lambda}_{-}(x)\left(\lambda_{+}(x+\mu)-\lambda_{+}(x-\mu)\right)
$$


where the phase $\eta_{\mu}(x)$ is the usual staggered quark phase given by

$$
\eta_{\mu}(x)=(-1)^{\sum_{i=1}^{\mu-1} x_{i}}
$$

Since the fields $\lambda$ and $\psi$ in the action are uncoupled we can take them to transform in different representations of one or more internal symmetry groups. For example,

$$
\bar{\psi}_{+} \rightarrow \bar{\psi}_{+} G^{\dagger}, \quad \psi_{-} \rightarrow G \psi_{-}, \quad \bar{\lambda}_{-} \rightarrow \bar{\lambda}_{-} H^{\dagger}, \quad \lambda_{+} \rightarrow H \lambda_{+}
$$

where $G \in S U(N)$ and $H \in S U(M)$. Notice that these symmetries have a vector character in the staggered lattice theory. These symmetries can be made local by inserting appropriate gauge links between the $\psi$ and $\lambda$ fields on neighboring sites. However, notice that unless $G=H$, it is then impossible to write down a single site mass term that preserves these symmetries. The usual staggered mass term

$$
\bar{\psi}_{+}(x) \lambda_{+}(x)+\bar{\lambda}_{-}(x) \psi_{-}(x)
$$

is not invariant. Throughout the remainder of this paper we will assume that the interactions factorize into a strongly coupled sector and a weakly coupled sector and that the $\psi$ and $\lambda$ fields transform differently only under the weak symmetries. Indeed, in what follows, we will suppress all indices related to the strong interactions. In addition, in this talk, we will restrict ourselves to the case where $G=S U(2)$ and $H$ is trivial. Explicitly we take the $\psi$ field to transform in the fundamental of $S U(2)$ while the $\lambda$ field is sterile. Both will also transform in the fundamental representation of a additional strongly coupled $S U(3)$ gauge interaction.

Within this class of models it is possible to write down a gauge invariant lattice four fermion term:

$$
\sum_{\mu} \phi_{+}^{\dagger}(x) U_{\mu}(x) \phi_{-}(x)
$$

where the composite Higgs field $\phi(x)$ (an $S U(3)$ singlet) is given by

$$
\phi_{+}^{\dagger}(x)=\bar{\psi}_{+}(x) \lambda_{+}(x), \quad \phi_{-}(x)=\bar{\lambda}_{-}(x) \psi_{-}(x)
$$

and the weak gauge link $U_{\mu}$ is needed to render the expression (weak) gauge invariant. If a condensate of $\phi^{\dagger} \phi$ develops it will signal the appearance of a lattice phase in which the weak $S U(2)$ interaction is completely Higgsed.

\section{Details}

We can look for such a condensate using numerical simulations. The gauging of the lattice kinetic term is given explicitly as

$$
\begin{aligned}
S_{K}= & \sum_{x, \mu} \bar{\psi}_{+}(x)\left(U_{\mu}(x) V_{\mu}(x) \psi_{-}(x+\mu)-U_{\mu}^{\dagger}(x-\mu) V_{\mu}^{\dagger}(x-\mu) \psi_{-}(x-\mu)\right) \\
& \sum_{x, \mu} \bar{\lambda}_{-}(x)\left(V_{\mu}(x) \lambda_{+}(x+\mu)-V_{\mu}^{\dagger}(x-\mu) \lambda_{+}(x-\mu)\right),
\end{aligned}
$$




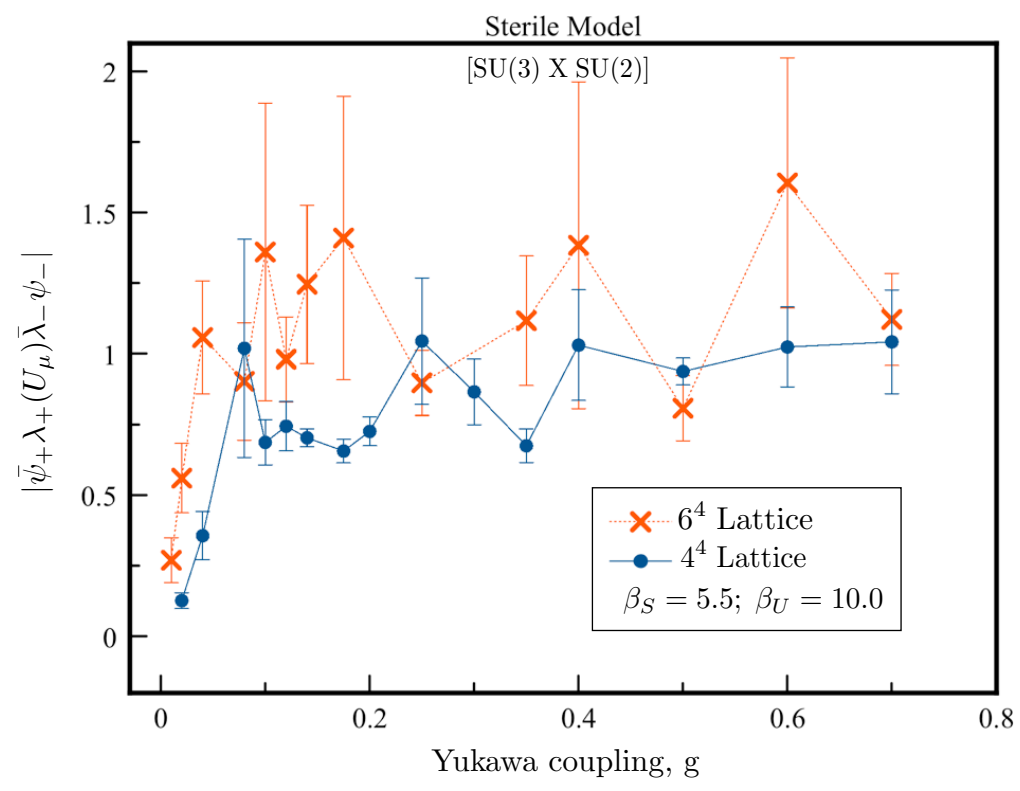

Figure 1: Absolute value of the four fermion condensate vs Yukawa coupling $g$

where, $V_{\mu}(x)$ and $U_{\mu}(x)$ correspond to the strongly coupled $\mathrm{SU}(3)$ and the weakly coupled SU(2) gauge groups respectively. In order to see the Higgs phase, we need to add a small perturbation to the above action via an auxiliary field $\phi$.

$$
\delta S=g \sum_{x}\left(\phi(x) \bar{\psi}_{+}(x) \lambda_{+}(x)+\phi^{\dagger}(x) \bar{\lambda}_{-}(x) \psi_{-}(x)\right)
$$

This field $\phi(x)$ is a local field which is needed to couple the two reduced fermions together and must transform under the gauge symmetry as a fundamental of $S U(2)$ in order that the perturbation is gauge invariant. To render the path integral well defined after integration over $\phi(x)$ one must then also add a suitable action for $\phi(x)$. We choose an additional simple term $\sum_{x} \phi^{\dagger}(x) \phi(x)$. The effect of these Yukawa terms is to add a small gauge invariant four fermion interaction to the action that favors the conjectured symmetry breaking pattern. The lattice action will also contain Wilson plaquette terms for both the $S U(3)$ and $S U(2)$ gauge fields with corresponding gauge couplings $\beta_{S}$ and $\beta_{W}$.

\section{Results}

It will be important in our argument to show that the magnitude of any induced four fermion condensate is insensitive to the value of this auxiliary Yukawa coupling $g$ and depends instead only on the magnitude of the strong gauge coupling.

Figure 1. shows a plot of the (absolute value of) the four fermion condensate as a function of the Yukawa coupling $g$ for fixed $\beta_{S}=5.5$ (the latter was determined to lie within the confining phase of the strong interaction for small lattices). We see that indeed a plateau develops and the 


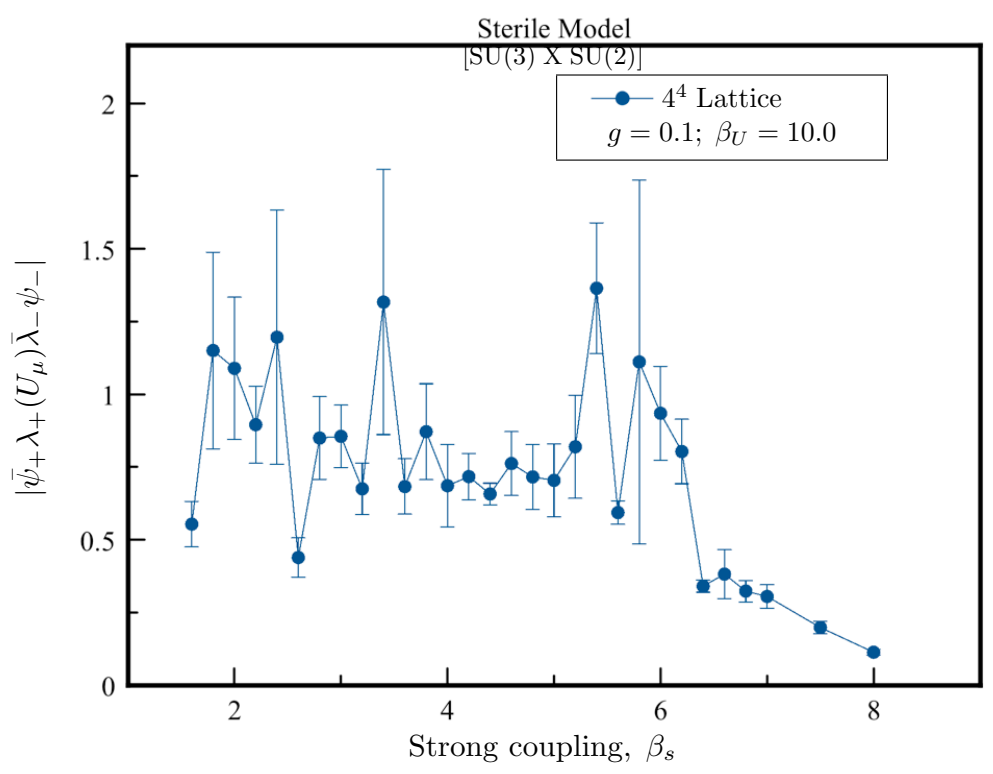

Figure 2: Absolute value of the four fermion condensate vs $\beta_{S}$

condensate is insensitive to the value of the Yukawa coupling over a wide range of $g$. In contrast figure 2. shows that the measured condensate does depend on the strong coupling constant $\beta_{S}$ (here $g=0.1$ is held fixed). The condensate is enhanced for small $\beta_{S}$ and falls towards zero once $\beta_{S} \geq 6.0$ which we observe is large enough to cause deconfinement in the strong sector. Most importantly we see no sign of a phase transition as we vary the strong coupling. This is evidence that any Higgs phase of the lattice theory may survive the continuum limit $\beta_{S} \rightarrow \infty$.

The appearance of a Higgs phase can be seen more clearly in figure 3 . which shows the Polyakov line corresponding to the weak gauge field as a function of the strong coupling $\beta_{S}$. For large $\beta_{S}$ the weak Polyakov line $P_{W}$ is large but as $\beta_{S}$ is lowered it rapidly crosses over to fluctuatate around a much smaller but non zero value. Since the weak Polyakov line $P_{W}=e^{-F T}$ measures the free energy $F$ of an isolated static quark in the fundamental representation of the weak gauge group a value approaching unity is associated with a deconfined phase. This is to be expected for a bare weak gauge coupling of $\beta_{W}=10.0$ on such a small lattice. Conversely, a confining phase typically would be associated with a small value of $P_{W}$. What is observed for small $\beta_{S}$ is intermediate between these two regimes and may be a signal of a Higgs phase. Furthermore, the crossover between these two regimes corresponds precisely to the switching on of the four fermion condensate and the observation of confinement in the strong sector. Thus our numerical results are at least consistent with the dynamical generation of a non-zero four fermion condensate and the appearance of a Higgs phase as a result of strongly coupled dynamics.

\section{Conclusions}

We have shown that lattice theories comprising two reduced staggered fermion fields can be 


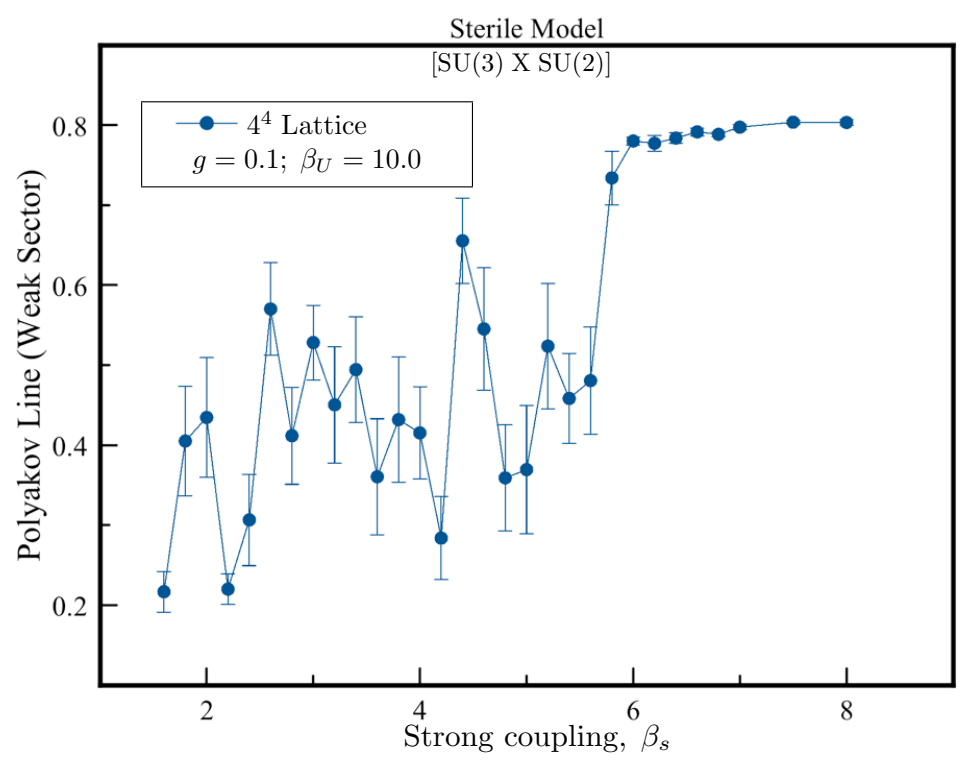

Figure 3: Weak Polyakov line versus $\beta_{S}$

constructed in such a way that they can naturally generate non-perturbative condensates that break gauge symmetries as a result of strongly coupled gauge dynamics. The key element that allows for symmetry breaking in these models is the absence of single site fermion bilinears which are invariant under these symmetries. In the case of gauge symmetries this means that the first gauge invariant object that can condense is a four fermion operator.

To understand these results it is instructive to imagine switching off the weak $S U(2)$ interaction completely. One can then recast the theory as a single conventional staggered fermion comprising the fields $\left(\psi^{1}, \lambda\right)$ together with an additional single reduced field $\psi^{2}$ where the explicit indices refer to the global $S U(2)$ symmetry. For sufficiently strong $S U(3)$ coupling one expects a single site condensate $\Sigma^{1}=<\bar{\psi}^{1}(x) \lambda(x)>$ to form. Such a condensate will spontaneously break the global $S U(2)$. Additionally, one expects that the remaining reduced staggered fermion will also form a condensate via a single link operator $\Sigma^{2}=<\bar{\psi}^{2}(x) U_{\mu}(x) V_{\mu}(x) \psi^{2}(x)>$. Now switch on the weak coupling. This cannot change the nature of the condensates that have already formed under the strong interaction (indeed this is we mean by weak coupling) so that one would conclude that the lattice theory will enter a phase in which the $S U(2)$ symmetry is Higgsed. The remaining question is whether this lattice Higgs phase survives the continuum limit.

At first glance this seems unlikely since the Vafa Witten theorem prohibits spontaneous breaking of vector symmetries. At finite lattice spacing this theorem does not apply since the fermion measure for this system of reduced staggered fermions is not positive definite. But the problem would seem to reassert itself for vanishing lattice spacing. However, in [6] we argue that the Vafa Witten theorem can be respected if this $S U(2)$ is reinterpreted as a broken axial symmetry in the continuum limit. This violates none of the usual theorems $[9,10]$ since the continuum theory is 
vector-like and trivially free of gauge anomalies.

Notice though that such a scenario would seem to imply that the vacuum state possesses less than the maximal flavor symmetry expected in the continuum theory ${ }^{1}$

In conclusion, it seems that the lattice models discussed here may serve as useful toy models for understanding the possibilities for dynamical symmetry breaking in strongly coupled gauge theories and can be used to test ideas such as the maximal attractive channel hypothesis and tumbling scenarios. In many respects the behavior of these theories mimics that of chiral fermions. It would be interesting to investigate such models via strong coupling expansions which would avoid possible sign problems.

\section{References}

[1] S. Weinberg, "Implications of dynamical symmetry breaking," Phys.Rev.D, vol. 13, p. 974, 1976.

[2] L. Suskind, "Dynamics of spontaneous symmetry breaking in the weinberg-salam theory," Phys.Rev.D, vol. 20, p. 2619, 1979.

[3] S. Raby, S. Dimopoulos and L. Susskind, "Tumbling Gauge Theories," Nucl. Phys. B 169, 373 (1980).

[4] H. Georgi and S. L. Glashow, "Unity of Elementary Particle Forces", Phys. Rev. Lett 32, 438 (1974)

[5] S. Elitzur, "Impossibility of Spontaneously Breaking Local Symmetries," Phys. Rev. D 12, 3978 (1975).

[6] S. Catterall and A. Veernala, "Dynamical Gauge Symmetry Breaking in Strongly Coupled Lattice Theories," [arXiv:1306.5668v2].

[7] C. V. den Doel and J. Smit, "Dynamical symmetry breaking in two flavor su(n) and so(n) lattice gauge theories," Nuclear Physics B, vol. 228, no. 1, pp. 122 - 144, 1983.

[8] M. F. L. Golterman and J. Smit, "Selfenergy and Flavor Interpretation of Staggered Fermions," Nucl. Phys. B 245, 61 (1984).

[9] H. Nielson and Ninomiya, " No go theorem for regularizing chiral fermions", Phys. Lett. B105, 219 (1981)

[10] L. H. Karsten and J. Smit, "Lattice Fermions: Species Doubling, Chiral Invariance, and the Triangle Anomaly,” Nucl. Phys. B 183, 103 (1981).

[11] Maarten Golterman and Yigal Shamir, private communication.

\footnotetext{
${ }^{1}$ Notice that it is possible that the system could retain maximal flavor symmetry together with an unbroken $S U(2)$ by the formation of gauge invariant one link condensates for each reduced staggered field. We see no evidence of this in our numerical work but it is possible that this could happen close to the continuum limit. Indeed, determining precisely how the $S U(2)$ symmetry is embedded into the global chiral group of the theory constitutes the famous vacuum alignment problem. We thank Maarten Golterman and Yigal Shamir for pointing this out [11]
} 